\title{
Queuing Theory Approach for Evaluating Rate of Transmission in Wireless Network Using Network Coding
}

\author{
Muhammed Zaharadeen Ahmed ${ }^{1}$, Othman Omran Khalifa ${ }^{1}$, Aisha Hassan \\ Abdallah Hashim ${ }^{1}$, Momoh. J. E. Salami ${ }^{2}$ and Muhanad Babikier ${ }^{2}$ \\ Department of Electrical and Computer Engineering, Faculty of Engineering, \\ International Islamic University Malaysia (IIUM), Malaysia \\ zaharadeen22@yahoo.com
}

\begin{abstract}
Due to the rapid development in the transmission of Wireless Network technologies, the need for rate evaluation using network coding demands urgent attention. This is solely dependent on how to improve on high performance communication channels for user's interest. In this paper, an approach of using queuing theory in a wireless network is presented. The idea of network coding in a wireless environment address the transmission of packets not only in unicast form but also address the conflict in packet movement across a channel. Network coding also contributes greatly in computer communication system and other computer based electronic appliances. This means that a source is transmitting packets to a particular destination according to the user's needs in a communication system. Packet transmission is enhanced within the wireless network using queuing theory. In this approach, the first routers are set to be the user access router and another directly connected router is set to be the source router. The user access router is set to connect 11 number of other routers where mainly the network coding is conducted. The results show an improvement in transmission rate by applying network coding as compared to other scenarios where networks coding are not utilized or the conventional routing either.
\end{abstract}

Keywords: Wireless, Router, Channel, Transmission rate, Network coding

\section{Introduction}

A wireless network is typically a network that has the ability to transmit over the air in a band of frequency such as the $2.4 \mathrm{GHz}$. Usually, a wireless local area network does not require aligning up of computer equipment for a line of sight transmission such as the Infrared Data Association also known as the (IDA). A wireless access points is also referred to as a base station. It connects to an Ethernet switch/hub/server and transmit a radio frequency signal for a wide coverage. The range covers hundred to thousand feet by infiltrating through the walls and other non-metallic barriers/shields. Any mobile host that connect wirelessly and continue to moves from one network point to another is being controlled. This control process is embedded in every access point known as the inter access point changeover [4]. 


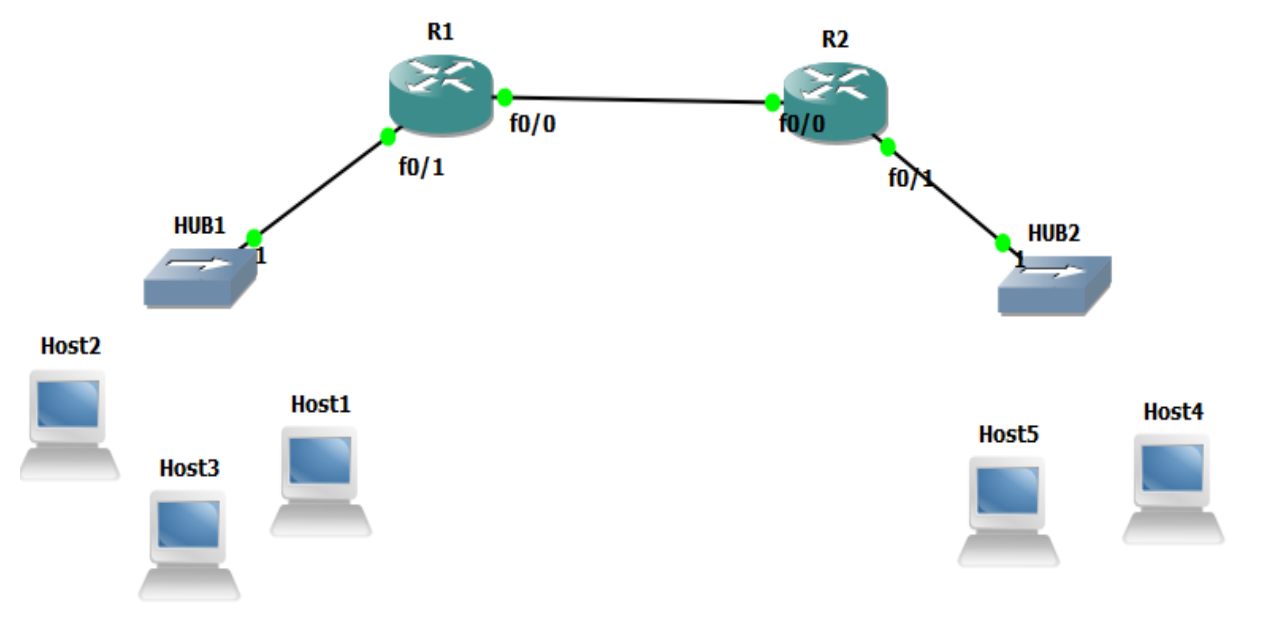

Figure 1. Hosts Connecting Wirelessly on a Network

It is obvious that computer systems and mobile phones uses wireless network cards. The manufacturers usually design the expansion in a redundant architecture. This is for reasons where the need to create a high speed work station may arise. Therefore, the processing capability, the power consumption and temperature regulation of a computer has to be put into consideration before

On the other hand, desktops and computer servers use socket cards or the plug in cards to connect to a computer network. Wireless LANs have some varieties of smaller forms sometimes called "Personal Area Networks", this is because their basic purpose is to typically serve an individual that is connecting a laptop computer or personal digital assistance to a desktop machine [1-2].

Packet transmission in network coding depends on packets forwarding between two router source and destination. The process will reliably ensure any missing packet is recovered and retransmitted to its respective destination. Also, the use of network coding addresses traffic in unicast form such that a source sends traffic to another destination. However, prior research examines other method of addressing traffic such as in multicast form. Multicasting is a process whereby an individual source transmits traffic to multiple destinations [3]. A source in network A intend to send message in multicast form. The message will locate the destination using an IP address and a MAC address of the layer three switch connecting network B. then the message will flood respectively. The figure below demonstrates the operation of network multicasting.

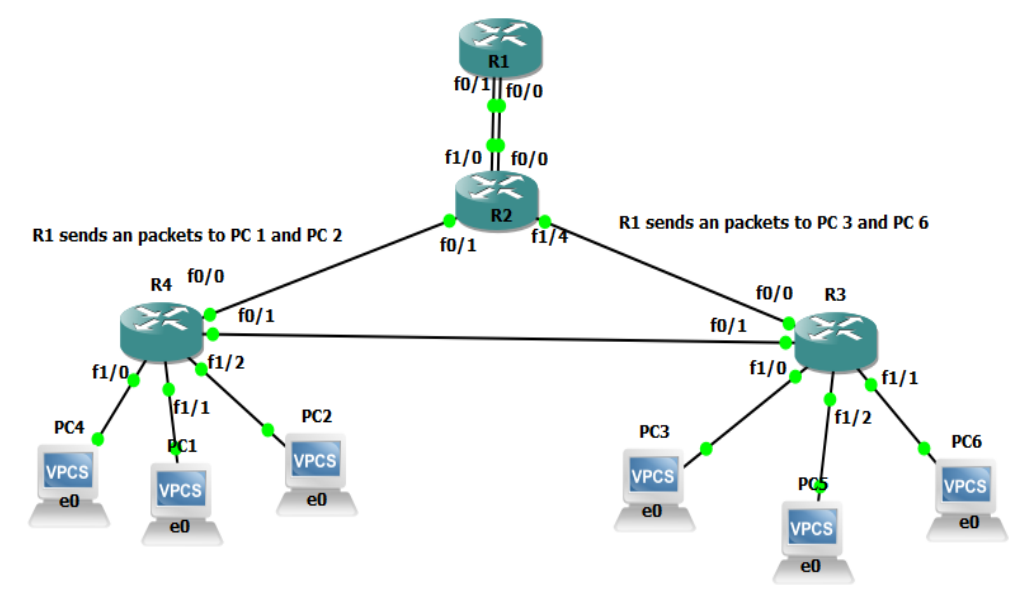

Figure 2. Multicast Packet Transmission [11] 


\section{Literature Review}

Nowadays, there has been a rapid and continuous development of using IEEE 82.11 wireless local area network in both home and small office networks. Cisco visual networking index clearly emphasize that, by the last quarter of the year 2019, a more substantial number of worldwide Internet protocol traffic will obviously be engendered by WAN devices.

However, [9] highlighted that by 2015 , almost $70 \%$ of all households across the globe that are now having a broadband access of Internet will acquire their personal wireless LAN base station. A high speed network requires regular upgrade so as to improve the network hardware, external attacks and routing policy. Different brands of network hardware can function together within the same communication domain [5].

When a wireless receiver is attached to an antenna with an infinitesimal noise amplification, the following parameters are observed. These are:

- One or more temporary period of frequency

- Unstable phase and

- A data repossession phase.

The negligible noise amplifier accepts an incoming radio frequency (RF) indicator through the antenna and amplifies them further [2].

Consequently, Quality of Service on a network is one of an essential parameter which include the rate of transmission and delay. These are hence regarded as the most essential considerations for end users. Wireless fidelity (WiFi) is regarded as a guarantee mark for apparatus based on a various set of the IEEE benchmarks, subsequently after the 802.11 working group wireless LANs.

Nowadays the fastest rate of transfer of data in the technology of computers is being taking care of by the fidelity Ethernet local area relationship. However, by means of consuming LAN in today's computers, a determination of implementing several technologies is highly possible now and in the near future [5].

\section{Wireless LANs with TCP and UDP Traffic Flows}

Considering the IEEE 802.11 technology as one of the major player the world market nowadays, network engineers continue to patronize the product as a result of its different high-speed versions. The IEEE802.11 technology have several varieties which includes 802.11e and the 802.11n standards [8]. However, the new market chances are being harnessed like the 802.11 metro scale networks.

The rapid expansion of the large number of wireless clients and the need for higher speed/bandwidth wireless network, usually require policy mechanisms to advance the media access control layer performance. In this regard, it is important to design a high demanding model so as to illustrate the communication between the 802.11 MAC and the operation of the complex layer transport protocols [7].

However, research has shown that there are limited findings carried out in the investigation of the relationship between the collision prevention apparatus of the standard 802.11 media access control protocol. With the dynamics of the complex layer transport protocols, this literature review presents how an issue is handled such as from an analytical, simulation, and experimental point of view. A design of a Markov chain model in [7] was carried out so as to calculate the distribution of number access points in operation using the IEEE 802.11 standard.

Note that connection reliability is usually relied upon a wired network setup. This is because traffic is guided from one connection source to the destination. When signal degradation occurs suddenly, packet flow continues transmission until the connection can 
no longer carry traffic. The figure below shows a description of traffic flow between UDP and TCP flow of traffic.

Based on the operation of transport control protocol, when encapsulated messages are transmitted, it ensures that the destination has to receive all the data chunks.

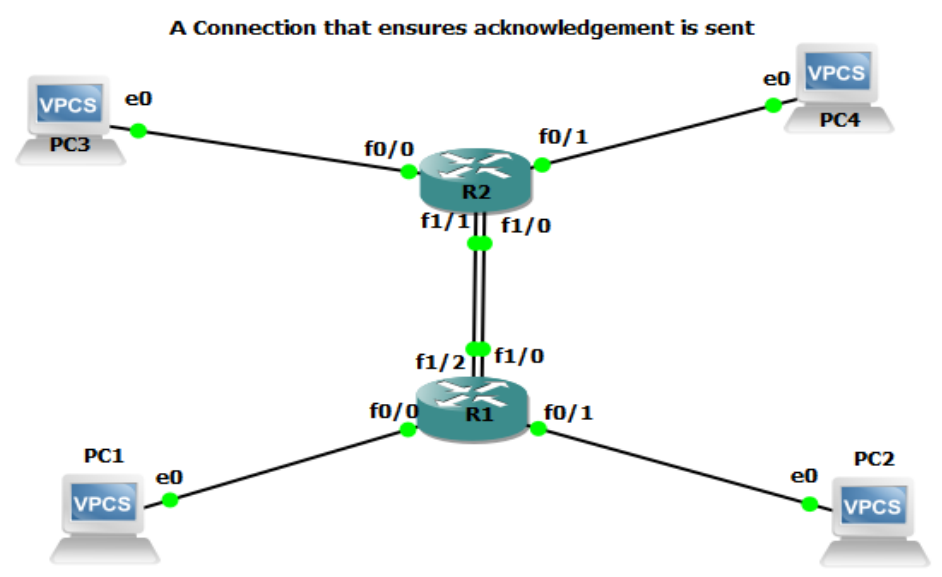

Figure 3. Transport Control Protocol [11]

While based on the operation of user datagram protocol, when encapsulated messages are transmitted, it does not require to fully ensure that all the chunks are transmitted to its destination [11].

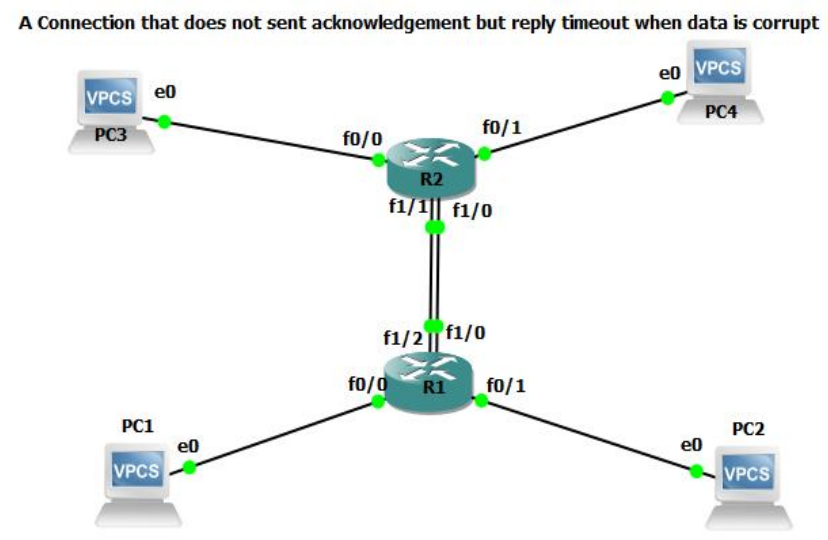

Figure 4. User Datagram Protocol [11]

An article in [9] illustrates an architecture that enhance data transmission rate. All required policies are documented on how to improve transmission rate on a data network. More research is required on the study of IEEE 802.11 standard so as to investigate the performance comparison with other standards. This is projected by the quality of service IEEE 802.11 Task Group E. The target of the 802.11e draft benchmark is to increase the quality of service (QoS) of wireless local area network because it may be employed to move real-time or concurrent media, (e.g., video and voice) with no delay from the "best effort" service level offered by contemporary 802.11 benchmark modelled as data-centric.

The table below presents some of the related literature reviews that was used in achieving this paper. 
Table 1. Reviews of Related Literature

\begin{tabular}{|c|c|c|c|}
\hline Research Topic & Approach & Strength & Limitation \\
\hline $\begin{array}{l}\text { Throughput Analysis } \\
\text { of WLAN with } \\
\text { Network Coding } 2013\end{array}$ & Simulation & $\begin{array}{l}\text { This paper shows } \\
\text { that using network } \\
\text { coding in WLAN } \\
\text { improvement in } \\
\text { throughput is by } 5 \text { to } \\
7 \% \text { by comparing to } \\
\text { routing without } \\
\text { network coding }\end{array}$ & $\begin{array}{l}\text { The throughput } \\
\text { needs to be } \\
\text { increased to more } \\
\text { than } 7 \% \text { using } \\
\text { network coding }\end{array}$ \\
\hline $\begin{array}{l}\text { Performance of } \\
\text { Wireless Network } \\
\text { using Network Coding } \\
\text { over Store and Forward } \\
\text { Technique } 2014\end{array}$ & simulation & $\begin{array}{l}\text { This paper presents } \\
\text { network coding } \\
\text { procedure at } \\
\text { transmission level in } \\
\text { wireless environment } \\
\text { only by XOR. }\end{array}$ & $\begin{array}{l}\text { The paper could not } \\
\text { examine the } \\
\text { implementation of } \\
\text { wireless network } \\
\text { that comprise } \\
\text { several nodes and } \\
\text { fetch out the } \\
\text { advantages of } \\
\text { network coding } \\
\text { compared networks } \\
\text { without network } \\
\text { coding. }\end{array}$ \\
\hline $\begin{array}{l}\text { Is rate adaptation } \\
\text { beneficial for inter } \\
\text { session network } \\
\text { coding? } 2008\end{array}$ & Simulation & $\begin{array}{l}\text { This paper } \\
\text { deliberates the } \\
\text { interaction between } \\
\text { Rate adaptation and } \\
\text { intersession network } \\
\text { Coding increase in } \\
\text { either wireless ad } \\
\text { hoc or mesh } \\
\text { networks }\end{array}$ & $\begin{array}{l}\text { The paper could not } \\
\text { proceed to increase } \\
\text { the network coding } \\
\text { gain at the terminal } \\
\text { node, }\end{array}$ \\
\hline $\begin{array}{l}\text { IEEE802.11n:Enhance } \\
\text { ments for Higher } \\
\text { Throughput in Wireless } \\
\text { LANS } 2005\end{array}$ & Simulation & $\begin{array}{l}\text { This paper } \\
\text { categorized frame } \\
\text { accumulation } \\
\text { instruments into } \\
\text { various and } \\
\text { orthogonal pieces } \\
\text { e.gs uplink against } \\
\text { downlink, centrally } \\
\text { coordinated against } \\
\text { distributed }\end{array}$ & $\begin{array}{l}\text { Various models of } \\
\text { throughput } \\
\text { enhancement were } \\
\text { proposed, some } \\
\text { were implemented } \\
\text { and some were not }\end{array}$ \\
\hline $\begin{array}{l}\text { A Wireless Test Bed } \\
\text { for Mobile } 802.11 \text { and } \\
\text { Beyond } 2006\end{array}$ & Analytical & $\begin{array}{l}\text { The paper reviewed } \\
\text { the Influence of } \\
\text { mobility wireless } \\
\text { system throughput } \\
\text { for mobile trains } \\
\text { with dissimilar speed }\end{array}$ & $\begin{array}{l}\text { The idea of the } \\
\text { model used in the } \\
\text { paper is not yet } \\
\text { implemented until } \\
\text { available } \\
\text { parameters are } \\
\text { presented. }\end{array}$ \\
\hline $\begin{array}{l}\text { Handoff Performance } \\
\text { Analysis of } \\
\text { Multihoming-based } \\
\text { Network Mobility }\end{array}$ & Simulation & $\begin{array}{l}\text { Provides } \\
\text { comparative } \\
\text { evaluation of } \\
\text { Multihoming based }\end{array}$ & $\begin{array}{l}\text { The paper could } \\
\text { not be able to } \\
\text { propose a model } \\
\text { for Combined }\end{array}$ \\
\hline
\end{tabular}




\begin{tabular}{|c|c|c|c|}
\hline Scheme (2016) & & $\begin{array}{l}\text { scheme in a mobile } \\
\text { network } \\
\text { environment and } \\
\text { also critical look at } \\
\text { the performance } \\
\text { comparison of two } \\
\text { schemes which are } \\
\text { mainly NEMO } \\
\text { BSP }\end{array}$ & $\begin{array}{l}\text { mechanisms for } \\
\text { link selection, but } \\
\text { was suggested as } \\
\text { a future work. } \\
\text { This will lead to } \\
\text { the ability to } \\
\text { handle with a } \\
\text { larger set of } \\
\text { Wireless access } \\
\text { technologies. }\end{array}$ \\
\hline $\begin{array}{l}\text { A Comparative Study } \\
\text { of IGP and } \\
\text { EGP Routing } \\
\text { Protocols, } \\
\text { Performance } \\
\text { Evaluation along } \\
\text { Load Balancing and } \\
\text { Redundancy across } \\
\text { Different AS (2016) }\end{array}$ & Simulation & 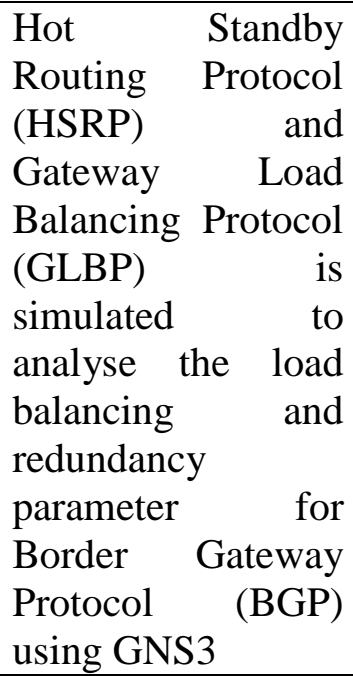 & $\begin{array}{l}\text { The Paper could } \\
\text { not be able to } \\
\text { Simulate network } \\
\text { with a much } \\
\text { larger traffic } \\
\text { volume such as } \\
\text { Multihoming } \\
\text { between the two } \\
\text { ISPs. }\end{array}$ \\
\hline
\end{tabular}

\section{Simulation Setup}

Simulation is carried out using various topologies. Below is an IP network, that simulates using the concept of queuing theory. Packet arrive and stored in a buffer before moving forward to the router's interface.

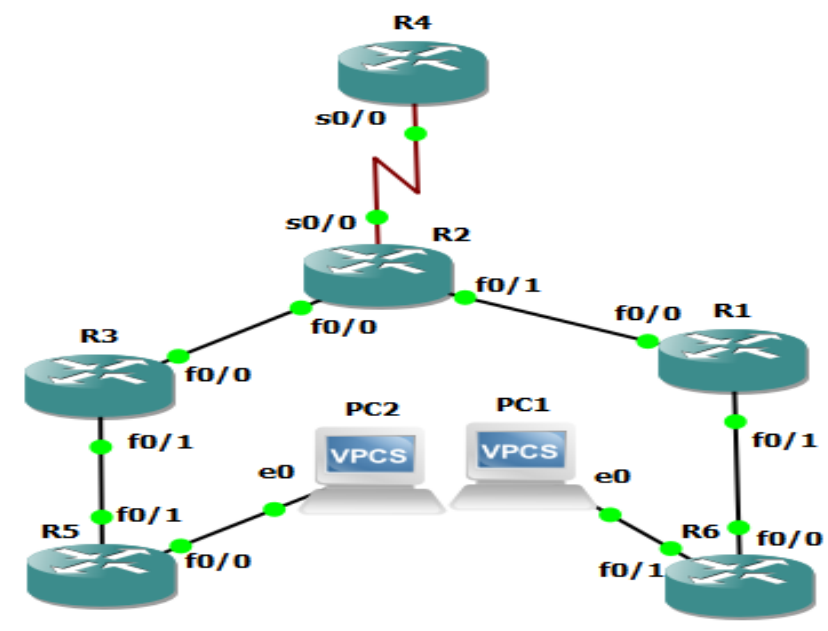

Figur 5. Packet Movement in a LAN Setup 
In this paper, two approaches are used in achieving the simulation setup. These are the conventional routing and network coding. The transmission rate in wireless network environment is considerably improve as compared to other literatures.

A router in a wireless local area network (WLAN) setup has the ability to encode received packets in multiple chunks so as to form a single encoded packet. The packet then is generated and being transmitted or spread out as a broadcast to the next directly connecting router in unicast form. This approach of transmission is done using network coding. However, using queuing theory, when packet arrive towards an interface of router, it is stored in a buffer as the first arriving packet, then moves out of the router to another interface for successful transmission to its destination. Delay is also recorded while the packet is being serviced. Simulations are carried out so as to determine the maximum rate of transmission on our network setup.
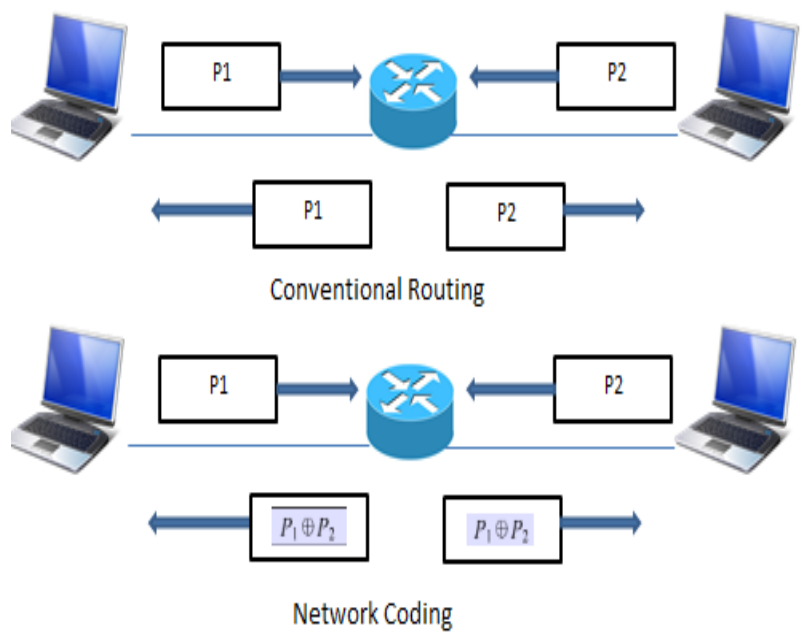

Figure 6. Node $A$ and $B$ Exchanging Packet $P_{1}$ and $P_{2}[1]$

In this simulation setup, packets movement are set to traverse between connecting routers according to different open model of queuing. A policy on restriction is places that, the total sum of the arrival rate at a particular router is less than or equal to the departure rate from that router. Mathematical formulas are used to actualize this analysis.

Stable rate of transmission is defined as the greatest possible quantity of packet generation at which the packet attain their respective destination with minimal predictable delay [1].

The model below describes how packet flows based on the model of queuing theory principle.

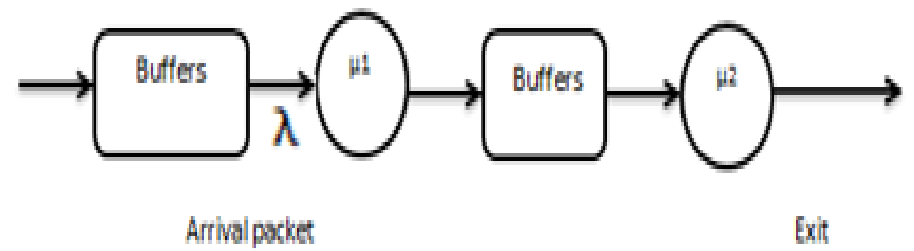

Figure 7. Structure of Queueing Process Based on Probalilities of Different States. [10]

In this wireless network, 11 router were put into consideration. Routers are labelled starting from the source router or terminal router denoted as I, and for all $i=1,2, \ldots, 10$. 
The normal range of transmission between each of the routers is one hop away. Any router beyond one hop away transmitting a unicast packet should be considered as a gateway by the access router ' $R$ ' (Relay node).

In a situation whereby a router is transmit a packet at a particular time, it cannot receive another packet further until after another period (session). The arrival rate of packet or information is denoted by the symbol of $\lambda$ which throughout is consistently allocated in the model of this wireless network. The throughput of the wireless network, the loss of packet and other network persuaded errors can be conveniently analyzed when packet flow is classified in the wireless network as below.

The figure below highlights the representation of the parameters used in the analysis.

\begin{tabular}{|c|c|}
\hline \\
\hline \multicolumn{2}{|c|}{$\begin{array}{l}\mathrm{P}_{\mathrm{t}}=(2 / 1+\mathrm{W}) \text { Represents the transmission likelihood at } \\
\text { represent contention Window })\end{array}$} \\
\hline \multicolumn{2}{|c|}{$\mathrm{P}_{t}^{\prime}=\left(2 / 1+\mathrm{W}^{\prime}\right)$ Represents the transmission likelihood at base station. } \\
\hline \multirow{2}{*}{\multicolumn{2}{|c|}{$\begin{array}{ll}\mu \text { and } \mu & \text { Represents the rate of serv } \\
\varrho \text { and } \rho ' & \text { Represents the amount of } t\end{array}$}} \\
\hline & ation \\
\hline \multicolumn{2}{|l|}{$\lambda$} \\
\hline \multirow{2}{*}{\multicolumn{2}{|c|}{$\begin{array}{l}a_{\mathrm{d} 1} \text { Present the rate of arrival at the terminal router which is destined to the neighbor } \\
a_{\mathrm{d} 2} \text { Represent the rate of arrival at the terminal router which is destined to the access } \\
\text { router }\end{array}$}} \\
\hline & \\
\hline \multirow{2}{*}{\multicolumn{2}{|c|}{$\begin{array}{l}\alpha_{\text {I }} \text { Represents the rate of arrival at the terminal router which is destined to its terminal } \\
\text { node and to be forwarded by the access node }\end{array}$}} \\
\hline & \\
\hline & \\
\hline
\end{tabular}

Figure 8. Parameters Used and their Definitions [1]

We use direct flow 1 which is given as D1 to be defined as the user access router has no activity. This means that, it await for packet to start flowing through any of its interfaces. We also use direct flow 2 which is given as D2 to be defined as the source router to user access router. Finally, we also use the indirect flow to de given as I. this is defined as the source router to the source router through the user access router. The analysis is further expressed using mathematical formulas as follows.

$$
\begin{aligned}
& \alpha \text { flow }=\text { rate of arrival of new packet }+\alpha \text { flow } \mathrm{p} \text { (node delivery failure) } \\
& \alpha \text { flow type }=\frac{\text { arrival packet }}{1-p(\text { node delivery failure })} \\
& \alpha \text { flow type }=\frac{\text { arrival packet }}{1-p(\text { nodedelivery failure })} \\
& \alpha_{\text {Flow }}=\text { rate of arrival of new packet }+. \alpha_{\text {Flow }} \mathrm{p} \text { (node delivery failure ) } \\
& \alpha \text { Flow type }=\frac{\text { arrival packetrate }}{1-p \text { nodedelivery failure }} \\
& \alpha \text { Flow type }=\frac{\text { arrival packetrate }}{\text { (packet delivery successfully) }}
\end{aligned}
$$


By applying the principle of queuing theory that at any node within the configuration, the arrival rate can be obtained from equation (6) above as

$$
\begin{aligned}
\alpha D 1 & =\frac{\lambda}{6(1-P t \rho)^{2}(1-P t t \rho l)(1-p l)} \\
\alpha D 2 & =\frac{\lambda}{6(1-P t \rho)^{5}(1-P t t \rho l)(1-p l)}
\end{aligned}
$$

Where the maximum rate of transmission or throughput is given by

$$
\lambda_{\mathrm{CR}}=\frac{\mu \rho \rho r\left(1-p_{t \rho)^{\mathrm{g}}}(1-p l)\right]}{3}
$$

Now, the complete intensity of traffic and the maximum rate of transmission or throughput at all the access nodes by utilizing network coding theory is to be expressed below as.

$$
\begin{aligned}
& \rho N C^{\prime} 2=\frac{\lambda\left[413-216(1-P \rho)^{\mathrm{g}}(1-p l)\right]}{72(1-P \rho)^{\mathrm{g}}(1-p l)\left[2-(1-P \rho)^{\mathrm{g}}(1-p l)\right]} \\
& \lambda N C 2=\frac{72 \mu \rho p^{\prime} z\left(1-p_{t}\right)(13-p l)\left[2-\left(1-p_{t p}\right)(13-p l)\right]}{[413-216(1-P t \rho)(1-p l)]}
\end{aligned}
$$

Note that, traffic at the terminal router and the base station are maintained to be $\left(\rho \leq 1\right.$ and $\left.\rho^{\prime} \leq 1\right)$.

\section{Result Analysis}

Results of this analysis illustrates that contention window at the access router was constant and to be exactly equal to the contention window at the terminal router. Hence the Performance comparison can be deduced using the formula below as.

$$
\alpha I=\alpha D 2
$$

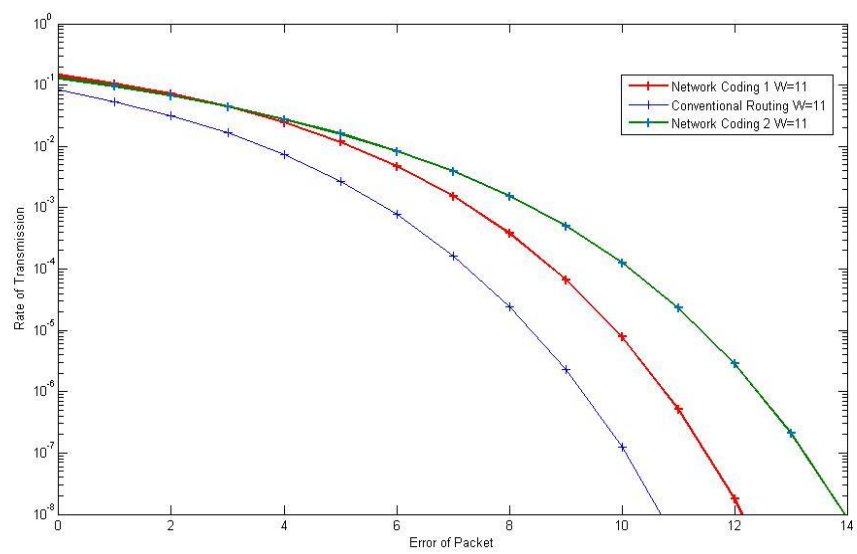

Figure 9. Rate of Transmission against Error of Packet in the Channel

Furthermore, the total rate of arrival rate at the terminal node are as follows; $2 \alpha \alpha \alpha D 1$ +3 $D 2+I$. The wireless local arear network performance with network coding by means of equation (7-11), the overall traffic intensity at the terminal nodes can be shown to be as follows: 


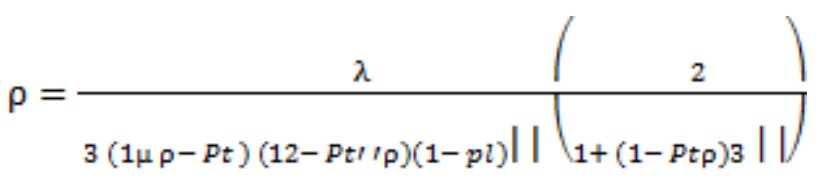

Note that, our scenario does not contain network coding at the terminal nodes. Moving on to the user access nodes, it can be easily seen that the overall traffic intensity without network coding is as follows

$$
\rho^{\prime}=\frac{3 \lambda}{\mu(1-P p)^{n} 3(1-p l)}
$$

However, with the use of network coding in our model, a more stability in the rate of transmission against the error of packet is realized. Graphs were plotted so as to observe the network performance. This was achieved by setting all the values driven from the formulas above of contention window indices with respect to time.

In this paper, it is observed that in network scenario where network coding is employed, a more performance is obtained in the rate of transmission with negligible amount of packet errors. This means that network coding plays a vital role as far as network setup is concer.

\section{Result Comparison}

The result obtained from our analysis is to be compared with a specific literature [1]. The transmission rate in the wireless network using 11 number of routers shows an improvement to the wireless network using less number of routers. Conventional routing shows good network performance both in this analysis and in [1]. Adding network coding makes it more accurate in performance.

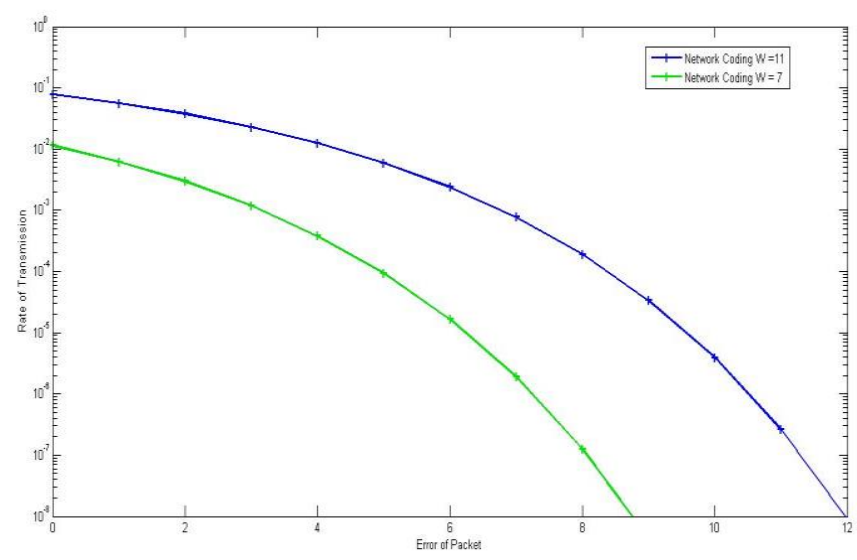

Figure 10. Result Comparison

\section{Conclusion}

This paper exploits the use of queuing theory approach to investigate the maximum rate of transmission for successful packet delivery from source to destination. The benefits of using network coding especially in a wireless environment was vividly brought to light. Not only packet collision in a network brought about packet misses. Link or channel error also plays a very crucial role. The major contributions of this analysis is that network performance is increased rapidly when analyzing a network using network coding. Also, the introduction of queuing theory approach makes the analysis more 
feasible mathematically. The initial router was set to be the user access router and another directly connected router was set to be the source router. The user access router was set to connect 11 number of other routers where mainly the network coding is conducted. The results show an improvement in transmission rate by applying network coding as compared to other scenarios where networks coding are not utilized or the conventional routing either. Analysis with more number of routers using various network parameters is to be considered as our future work

\section{References}

[1] P. R. Mane, M. Sathish Kumar, G. Sudhakar and G. Prabhu, "Throughput Analysis of WLAN with Network Coding", International Journal of Future Computer and Communication, vol. 2, no. 1, (2013), 23.

[2] Computer Desktop Encyclopedia. (c) 1981-2001. The Computer Language Company Inc. All rights reserved. Ver. 14.3m, 3rd Quarter 2001

[3] Y. Kim and G. De Veciana, "Is rate adaptation beneficial for inter-session network coding?", In MILCOM 2008-2008 IEEE Military Communications Conference on IEEE, (2008), pp. 1-7.

[4] P. Gawłowicz, S. Zehl, A. Zubow and A. Wolisz, "NxWLAN: Neighborhood eXtensible WLAN", arXiv preprint arXiv:1607.03254, (2016).

[5] I. Salem Al-Safe Abdullah, A. Amin Babiker and N. Mustafa, "The Impact of Distance on WLAN and LAN Network Performance".

[6] M. Hempel, H. Sharif, T. Zhou and P. Mahasukhon, "A wireless test bed for mobile 802.11 and beyond", In Proceedings of the 2006 international conference on Wireless communications and mobile computing, ACM, (2006), pp. 1003-1008.

[7] R. Bruno, M. Conti and E. Gregori, "Throughput analysis and measurements in IEEE 802.11 WLANs with TCP and UDP traffic flows", IEEE Transactions on Mobile Computing, vol. 7, no. 2, (2008), pp. 171-186.

[8] Y. Xiao, "IEEE $802.11 \mathrm{n}$ : enhancements for higher throughput in wireless LANs", IEEE Wireless Communications, vol. 12, no. 6, (2005), pp. 82-91.

[9] Y. Solomon, "Defining and improving data throughput in wireless LAN", Texas Instruments White Paper, (2003).

[10] A. Melikov and A. Rustamov, "Queuing management in wireless sensor networks for QoS measurement", Wireless Sensor Network, vol. 4, no. 9, (2012), pp. 211.

[11] J. Kurose and K. Ross, “Computer Networking: A Top Down Approach”, (2012). 
International Journal of Future Generation Communication and Networking Vol. 10, No. 6 (2017) 